\title{
BMJ Open Improved diagnostics of infectious diseases in emergency departments: a protocol of a multifaceted multicentre diagnostic study
}

\author{
Helene Skjøt-Arkil (D , , ${ }^{1,2}$ Anne Heltborg, ${ }^{2,3}$ Morten Hjarnø Lorentzen, ${ }^{1,2}$ \\ Mariana Bichuette Cartuliares, ${ }^{1,2}$ Mathias Amdi Hertz (D) , ,,5 Ole Graumann, 5,6 \\ Flemming S Rosenvinge, ${ }^{7}$ Eva Rabing Brix Petersen, ${ }^{8}$ Claus Østergaard, ${ }^{9}$ \\ Christian B Laursen, ${ }^{5,10}$ Thor Aage Skovsted, ${ }^{8}$ Stefan Posth, ${ }^{5,11}$ Ming Chen, ${ }^{2,12}$ \\ Christian Backer Mogensen (1) ${ }^{1,2}$
}

To cite: Skjøt-Arkil H, Heltborg A, Lorentzen MH, et al. Improved diagnostics of infectious diseases in emergency departments: a protocol of a multifaceted multicentre diagnostic study. BMJ Open 2021;11:e049606. doi:10.1136/ bmjopen-2021-049606

- Prepublication history and additional supplemental material for this paper are available online. To view these files, please visit the journal online (http://dx.doi.org/10.1136/ bmjopen-2021-049606)

Received 27 January 2021 Accepted 17 September 2021

Check for updates

(C) Author(s) (or their employer(s)) 2021. Re-use permitted under CC BY-NC. No commercial re-use. See rights and permissions. Published by BMJ.

For numbered affiliations see end of article.

Correspondence to Helene Skjøt-Arkil; Helene.Skjoet-Arkil@rsyd.dk

\section{ABSTRACT}

Background The major obstacle in prescribing an appropriate and targeted antibiotic treatment is insufficient knowledge concerning whether the patient has a bacterial infection, where the focus of infection is and which bacteria are the agents of the infection. A prerequisite for the appropriate use of antibiotics is timely access to accurate diagnostics such as point-of-care (POC) testing. The study aims to evaluate diagnostic tools and working methods that support a prompt and accurate diagnosis of hospitalised patients suspected of an acute infection. We will focus on the most common acute infections: community-acquired pneumonia (CAP) and acute pyelonephritis (APN). The objectives are to investigate (1) patient characteristics and treatment trajectory of the different acute infections, (2) diagnostic and prognostic accuracy of infection markers, (3) diagnostic accuracy of POC urine flow cytometry on diagnosing and excluding bacteriuria, (4) how effective the addition of POC analysis of sputum to the diagnostic set-up for CAP is on antibiotic prescriptions, (5) diagnostic accuracy of POC ultrasound and ultralow dose (ULD) computerized tomography (CT) on diagnosing CAP, (6) diagnostic accuracy of specialist ultrasound on diagnosing APN, (7) diagnostic accuracy of POC ultrasound in diagnosing hydronephrosis in patients suspected of APN.

Methods and analysis it is a multifaceted multicentre diagnostic study, including 1000 adults admitted with suspicion of an acute infection. Participants will, within the first 24 hours of admission, undergo additional diagnostic tests including infection markers, POC urine flow cytometry, POC analysis of sputum, POC and specialist ultrasound, and ULDCT. The primary reference standard is an assigned diagnosis determined by a panel of experts. Ethics, dissemination and registration Approved by Regional Committees on Health Research Ethics for Southern Denmark, Danish Data Protection Agency and clinicaltrials.gov. Results will be presented in peerreviewed journals, and positive, negative and inconclusive results will be published.

Trial registration numbers NCT04661085, NCT04681963, NCT04667195, NCT04652167,

\section{Strengths and limitations of this study}

- It is a pragmatic study that reflects reality and has potential for substantial clinical significance.

- The study combines diagnostics and knowledge from five different medical specialties.

- The study is complex and contains a number of substudies which share the same population.

- The study is only generalisable to settings with a similar technological context and trained staff.

- COVID-19 and the consequent societal lockdown might affect patient distribution.

NCT04686318, NCT04686292, NCT04651712, NCT04645030, NCT04651244.

\section{INTRODUCTION}

\section{Antibiotic resistance}

Multiresistant bacteria are one of the major threats to public health. ${ }^{1}$ The incidence of multiresistant bacteria is increasing in Denmark $^{2}$ and every 20th patient admitted to a Danish emergency department (ED) is colonised with multiresistant bacteria. ${ }^{3}$ Denmark has focused on this challenge ${ }^{4}$ by screening special patient groups for multiresistant bacteria, ${ }^{56}$ and by initiating campaigns to reduce antibiotic consumption-mainly the use of broad-spectrum antibiotics in hospitals. ${ }^{4}$

The Danish Ministry of Health has made extensive efforts targeting the use of antibiotics in hospitals. However, the major obstacle in reducing the prescription of broadspectrum antibiotics is insufficient knowledge concerning whether the patient has a bacterial infection, where the focus of infection is and which bacteria are the agents of the infection. ${ }^{8}$ 
Uncertainty in the answers to these three questions often leads a clinician to choose a broad-spectrum antibiotic at the onset of treatment. Unfortunately, the prescription of a broad-spectrum antibiotic is rarely revised when laboratory results are available, often because the patient already has been discharged. ${ }^{9}$

\section{Acute infections and diagnostic tools}

A prerequisite for appropriate use of antibiotics is timely access to accurate diagnostic tests, since treatment of acute infections should be initiated within a few hours to avoid serious complications such as bacteraemia, sepsis, organ failure, septic shock and death. ${ }^{10}$ The most common conditions among ED patients with suspected infections are community-acquired pneumonia (CAP) and acute pyelonephritis (APN). ${ }^{11}{ }^{12}$ Diagnosing CAP and APN can be challenging as symptoms are often weak and non-specific and the current methods for focal and aetiological diagnosis have low sensitivity and specificity and often deliver results after the decision regarding antibiotic treatment has been made. ${ }^{9} 1314$

The COVID-19 pandemic has highlighted the need of accurate diagnostic tests. Quick and correct classification of pneumonia as COVID-19, another viral or bacterial pneumonia, or even COVID-19 complicated with bacterial pneumonia, is of vital importance to select the correct treatment (including antibiotics), and the correct infection control measures, including isolation.

In order to make the correct diagnosis and prescribe an appropriate and targeted treatment within a few hours of admission, it is important to the physician to be able to answer the following three questions: (a) Is it an infection that requires antibiotic treatment (bacterial infection marker)?; (b) Where is the focus of infection (imaging diagnosis)?; (c) Which bacteria should the prescribed antibiotic target (aetiological diagnosis)?

\section{Bacterial infection markers}

To support the diagnosis of an infection and assess its severity, a measure of the systemic inflammatory response is useful, for example, abnormal temperature, elevated leucocyte count with neutrocytosis or elevated $\mathrm{C}$ reactive protein (CRP). Some uncertainty is associated with CRP because it has a delayed response to bacterial infection and often is elevated in non-infectious inflammatory conditions. ${ }^{15}$ A more sensitive and specific marker that can differentiate between bacterial and viral infection and reflect the severity of the infection is desired. ${ }^{16}$ Serum procalcitonin (PCT) has potential as a diagnostic tool in suspected bacterial infections ${ }^{17}$ and can distinguish between viral and bacterial pneumonias. ${ }^{18}$ Soluble urokinase plasminogen activator receptor (suPAR) might have a potential as a marker for acute bacterial infections requiring antibiotic treatment. ${ }^{19}$ However, there are no well-conducted studies which compare simultaneously all three biomarkers' diagnostic abilities for bacterial infections in general or in relation to CAP or APN. ${ }^{1620}$

\section{Imaging diagnostics}

The CAP diagnosis is primarily based on clinical symptoms and findings, supplemented with chest X-ray, which has a low sensitivity and specificity. ${ }^{21}$ Identifying an improved imaging alternative with high diagnostic sensitivity and specificity and minimal risk to the patient is imperative. Computerized tomography (CT) scans, for example, high-resolution CT (HRCT) provides a detailed diagnosis of thoracic diseases, but the radiation dose is high and potentially harmful. Low-dose CT has shown promising diagnostic results, but the radiation dose is still potentially harmful. ${ }^{22}$ Ultralow dose CT (ULDCT) of the thorax could be an alternative, but has yet to be studied within an ED context. Another relevant imaging modality is ultrasound scanning (US). US of the lungs is useful to diagnose pulmonary oedema and pleural effusion, but the value of US performed by a novice operator when diagnosing CAP in an ED setting needs further investigation. $^{23}$

Currently, no imaging methods are used to verify the diagnosis of APN. The diagnosis is primarily based on unspecific clinical findings, ${ }^{24}$ and is often not confirmed microbiologically. ${ }^{25}$ Complicating factors such as hydronephrosis and renal abscess can be visualised with conventional US. ${ }^{26}$ Contrast enhanced US (CEUS) seems to be a promising diagnostic imaging modality of acute renal inflammation. ${ }^{27} 28$ The value and suitability in a clinical setting of this more advanced US investigation is unknown.

\section{Aetiological diagnostics}

Sputum can be cultivated to determine the agent of CAP. However, results are often unspecific and not available until after discharge of the patient or completion of treatment. ${ }^{9}$ A point-of-care (POC) tool providing rapid microbiological results on, for example, sputum samples would therefore be useful. Systems are available today based on PCR methods with results available within 1 hour for a variety of viral and bacterial pathogens. ${ }^{29}$ The impact of such fast diagnostic systems on antibiotic prescriptions has not been investigated in an ED context.

The diagnosis of APN is verified by significant bacteriuria in urine culture, ${ }^{25}$ but as many as half of the patients with clinical APN fail to meet this diagnostic criterion. Unfortunately, the time from sample to result for urine cultures is more than 24 hours. ${ }^{24} 253031$ Urine test strips are unreliable with low specificity and low predictive values. $^{32}$ Therefore, a POC test is desired, which can provide rapid results and quickly identify a bacteriuria. One such tool may be urine flow cytometry (UFC), which has shown promising diagnostic value for the exclusion of bacteriuria with a high negative predictive value. ${ }^{33}$ However, better documentation for its use as an ED diagnostic screening method is needed.

\section{Aim and objectives}

Our broad hypothesis is that improved diagnostic strategies for patients in ED with suspicion of systemic infection can contribute to more rapid and accurate diagnosis. 
Therefore, we assume that a more appropriate antibiotic treatment can be administered to these patients.

The project aims to evaluate alternative diagnostic tools and working methods that support a prompt and accurate diagnosis of hospitalised patients suspected of an acute infection. We will focus on the most common ED infections: CAP and APN. The research objectives are to answer the following questions:

1. What are the patient characteristics and treatment trajectory of the different ED infections?

2. What is the diagnostic and prognostic accuracy of the infection markers suPAR and PCT in patients with suspected CAP and APN?

3. What is the diagnostic accuracy of POC-UFC on diagnosing and excluding bacteriuria?

4. How effective is the addition of POC-PCR analysis of sputum to the diagnostic set-up for CAP on antibiotic prescribing?

5. What is the diagnostic accuracy of POC-US and ULDCT on diagnosing CAP?

6. What is the diagnostic accuracy of CEUS on diagnosing APN?

7. What is the diagnostic accuracy of POC-US in diagnosing hydronephrosis in patients suspected of APN?

The ultimate goal is to combine the results of all these seven objectives into a novel diagnostic model which the ED physician can apply when receiving a patient with suspicion of infection.

\section{METHODS}

\section{Study design}

The study is designed as a multifaceted multicentre diagnostic study. Participants will undergo additional diagnostic tests depending on the primary suspected focus of infection.

The study protocol is reported in accordance with the Standard protocol items: Recommendations for interventional trials statement. ${ }^{34}$ Informed consent materials can be found in online supplemental appendix I, biological specimens in online supplemental appendix II, and schedule of enrolment, interventions, and assessments in online supplemental appendix III.

\section{Setting}

The study will recruit participants from three Danish EDs: the regional hospital, Lillebælt Hospital in Kolding, the regional hospital, Hospital Sønderjylland in Aabenraa, and the university hospital, Odense University Hospital in Odense. Enrolment commences from 8 February 2021 and continues until the predefined sample size has been reached.

Project assistants will recruit the participants and collect data. The project assistants will have a healthcare education (physicians, physiotherapists, nurses and medical students). They are certificated in focused US of kidney and lung (1 day POC-US course, 25 supervised scans and



Figure 1 Design of patient flow and diagnostic tracks. APN, acute pyelonephritis; CAP, community-acquired pneumonia; ED, emergency department: HRCT, high-resolution CT; POC, point-of-care; UFC, urine flow cytometry; ULDCT, ultralow dose CT; US, ultrasound scanning.

Objective Structured Assessment of US Skills test) within 1 month from enrolment.

The study originates from the Emergency Research Unit affiliated at University Hospital of Southern Denmark and Department of Regional Health Research at University of Southern Denmark.

\section{Population and eligibility criteria}

Inclusion of patients is based on the receiving ED physician's initial clinical assessment of the patient. Adults aged 18 years or older admitted to the ED will be invited to participate in the study, if the receiving physician suspects the patient is having an infection. Only patients able to give informed consent will be participating in the study. Depending on primary suspected focus of infection (CAP, APN or other/ unknown), the patients will be included into one of three diagnostic tracks (A, B or $\mathrm{C}$ ) as shown in figure 1 .

Exclusion criteria that apply to all three tracks at time of recruitment:

- If the attending physician considers that participation will delay a life-saving treatment or directly transfer to intensive care unit.

- Admission (defined as $>24$-hour hospital visit) within the last 14 days to avoid hospital-acquired infections.

- Verified COVID-19 within 14 days before admission to avoid a skewed population consisting of patients with COVID-19 instead of patients with CAP. Patients suspected of COVID-19, at the time of recruitment, 
will not be excluded-nor if subsequently tested positive.

- Pregnant women, this to uniform all the studies. At the participating EDs, the pregnant women represent a very small patient group, as they are admitted directly to the ward.

- Severe immunodeficiencies:

- Primary immunodeficiencies

- Secondary immunodeficiencies

- HIV positive, with a cluster of differentiation 4 cell count $<200$.

- Patients receiving immunosuppressive treatment (Anatomical Therapeutic Chemical classification L04A).

- Corticosteroid treatment ( $>20 \mathrm{mg} /$ day prednisone or equivalent for $>14$ days within the last 30 days).

- Chemotherapy within 30 days.

Exclusion criteria that only apply to patients with suspected CAP (track A):

- Patients <40 years old are excluded from the ULDCT and HRCT due to risk of cancer from radiation.

- Patients $<65$ years who already participated once will be excluded from ULDCT and HRCT due to risk of cancer from radiation.

Exclusion criteria that only apply to patients with suspected APN (track B):

- Patients are excluded from MRI according to common MRI exclusion criteria (eg, contraindicating metal in the body) and claustrophobia.

- Patients with known allergy to US contrast.

\section{Recruitment}

The study assistants will identify potential eligible patients through the local logistic system, which lists patients visiting the ED (Cetrea Anywhere), According to the local guidelines, a medical clinical assessment of the patients is performed within half an hour from arrival at the ED. ${ }^{35}$ The study assistant will, immediately after the assessment, consult the receiving physician to ask if (a) a systemic infection is suspected and (b) what the most likely focus is: lungs, urinary tract, elsewhere/unknown. If the patient meets the eligibility criteria, the study assistant will present the study both verbally and in writing, and invite the patient to participate in the study.

\section{Procedure}

The study assistant will, after obtaining written consent, order blood samples, urine sample and the diagnostic tests described in the assigned track. The study assistant will collect data for patient characteristic by looking in the patient record and by patient interview.

\section{Infection markers}

Blood samples will be collected by a medical laboratory technologist and transferred to the local laboratory for analysis of CRP (routine analysis), PCT and suPAR. Laboratory staff will be blinded to participant diagnosis and outcome. PCT results will be available to the treating physician, but the suPAR result will not be available. CRP will be measured using an immunoturbidimetric assay (Tina-quant, Roche) on Roche/Hitachi Cobas systems. Plasma PCT will be quantified by an automated sandwich immunoassay 'ECLIA' (Elecsys, BRAHMS PCT-analyses) on Cobas within 2 hours from collection according to standard procedure. Plasma suPAR will be quantified by using the commercial available suPARnostic Tubilatex assay reagents (ViroGates, Denmark) on Cobas as previously validated. ${ }^{36}$ Separated plasma is kept refrigerated and analysed for suPAR within 48 hours after collection.

\section{Point-of-care urine flow cytometry}

A urine sample will be collected according to routine procedure by the study assistant. The sample will be divided into three aliquots: one for routine urine culturing, one for routine dipstick analysis and one half for POC-UFC analysis (UF-5000, Sysmex, Kobe, Japan). The POC-UFC analysis will be performed according to manufacturer's instruction and conducted by study assistants or laboratory staff in a POC laboratory close to the department to which the transport time is less than 10 min. Laboratory staff will be blinded to participant diagnosis and outcome. The results of the POC-UFC analysis will not be visible to the treating physician.

The results of the dipstick analysis and the urine culturing will be available to the treating physician as part of the usual procedure (within 1 hour for dipstick and after up to several days for culturing).

\section{POC-PCR sputum analysis}

A sputum sample will be collected according to standard procedure as soon as possible after recruitment by the study assistant. This sample will be randomly assigned to one of two groups with 1:1 allocation: (1) POC-PCR analysis (Biofire FilmArray Pneumonia Panel plus, Biomérieux, Marcy l'Etoile, France) in accordance with manufacturer's instruction, ${ }^{37}$ and (2) routine microbiology analysis (culturing and PCR). Expectorated sputum or tracheal secretions will be used for the PCR analysis. All sputum samples will be cultured. Gram stain and microscopy are not included in the analysis.

The randomisation will be performed by the study assistants and generated electronically using Research Electronic Data Capture (REDCap) Randomization Module ${ }^{38}$ with permuting blocks and stratified according to sites. Allocation concealment is ensured, as randomisation is performed electronically and the study assistants administering the randomisation will not have access to the randomisation code. The allocation is revealed after consent is obtained and sputum collection successful.

The study assistants or laboratory staff will perform the POC-PCR analysis in a POC laboratory at the ED or close to the department to which the transport time is less than $10 \mathrm{~min}$. The used POC-PCR targets 27 of the most common pathogens involved in lower respiratory tract infections (online supplemental appendix IV). The result 
of the POC-PCR will be presented by the study assistant to the treating physician within 4 hours on admission. The treating physician will, along with the result, receive a recommended action list (online supplemental appendix V), developed by microbiologists.

The patients will be blinded, and the investigator will be blinded to data management and analysis. Outcome adjudicators will not be blinded.

\section{Point-of-care ultrasound scanning}

A POC-US (Butterfly iQ+, GM Medical) of the lungs will be performed bedside as focused lung US (FLUS) by study assistant within 24 hours after admission. FLUS is used to diagnose pneumothorax, pleural effusion and interstitial syndrome. Additionally, signs of pneumonia, that is, liverlike alveolar consolidation with shredded borders and air bronchograms will be described. Diagnostic criteria used are in accordance with international consensus. ${ }^{39} 40$ FLUS will be conducted immediately before or after the CT scans. The FLUS result will not be available to the treating physician unless the result requires immediate action (pneumothorax or large pleural effusions).

A POC-US (Butterfly iQ+) of the kidneys will be conducted bedside by a study assistant within 24 hours after admission in order to assess whether hydronephrosis is present or absent. If present, the condition will be graded in grades $1,2,3$ or $4 .{ }^{41}$ The result will not be available to the treating physician since the patient is examined by a radiologist immediately after, and the results from this examination are reported to the clinician according to standard care.

\section{ULDCT and HRCT}

The ULDCT and HRCT of the thorax scans are performed in the same scanning sequence, thus on the same scanner. A specially designed technical protocol is the basis of the ULDCT and will, prior to inclusion through a minor pilot study, be optimised at each site of inclusion to ensure uniform quality and dose. The radiological findings from ULDCT will be reported systematically using standardised assessment templates by radiologists. The HRCT will be performed according to standard protocols at each hospital, but only during inspiration to limit radiation dose. HRCT will be reference standard for FLUS and ULDCT and interpreted by lung expert radiologists. The reports from POC-US, ULDCT and HRCT, respectively, will be blinded. Study consultant radiologists with experience from ED patients will post-process report the ULDCT scans systematically using specially developed research report templates. The results of ULDCT and HRCT will be available to the treating physician within a week. If a result requires immediate action, the clinician will be contacted directly by the examiner (pneumothorax and large pleural effusions), according to standard care. If a participant is discharged before the scans have been performed, they will be offered the scan in an outpatient setting.
CEUS and MRI

A specialist US will be performed at the radiology department, including conventional greyscale US and CEUS with intravenous injection of $1.5 \mathrm{~mL}$ ultrasound contrast (Sonovue, Bracco). At the same time, or as close as possible, an MRI without intravenous contrast of the kidneys will be conducted. The MRI will include the following sequences: planning, Dixon, T1 mapping, T2, T2 mapping, apparent diffusion coefficient (ADC) (100, 400,800 ), MRI angio (3D VIBE) and phase contrast. The radiological findings will be described systematically using standardised assessment templates. The report from US and MRI, respectively, will be blinded. A renal expert radiologist will interpret the MRI and will post-process report the imaging systematically using specially developed research report templates. Imaging from the CEUS will be evaluated in an external post-processing software algorithm (Vuebox, Bracco). The non-experimental results of the scans will be available to the treating physician within a week. If a result requires immediate action (suspicion of pyonephrosis or renal abscess), the clinician will be contacted directly by the examiner, according to standard care. If a participant is discharged before the scans have been performed, they will be offered the scans in an outpatient setting.

\section{Expert panel reference standard}

Unless otherwise stated, the reference standard is the assigned diagnosis determined by a panel of experts. The panel consists of two consultants: a specialist in emergency medicine and a specialist in infectious medicine with considerable experience within acute infections. They will determine the final diagnosis based on all relevant information in medical records and study database available from the admission including routine blood analysis, blood/urine/sputum culturing, POC-PCR, routine and study imaging (including HRCT and MRI), and clinical information. The final diagnosis will be based on information available within the first week after admission. A standardised template in REDCap will be used (online supplemental appendix VI), and the experts will register if the patient has an infectious disease, if the focus of infection is the lungs, kidneys or other, and specify the infection by adding an international classification of diseases (ICD-10 diagnosis code). If the patient has two focal diagnoses, for example, pneumonia and APN, the assessment will be based on what is the most probable cause of infection on admission. Conflicts will be discussed until consensus is reached. In this study, we define APN as a urinary tract infection with typical local symptoms and systemic affection (ie, fever, sepsis), thus indicating ascension of infection above the bladder.

\section{Data collection and management}

All data will be collected in REDCap. Data will be pseudoanonymised and managed and analysed using STATA or $\mathrm{R}$ in collaboration with a biostatistician. 
For each participant, information on predefined clinical parameters on arrival will be obtained from the medical record including symptoms, lifestyle factor signs, disease severity, vital parameters, triage at arrival, comorbidities, functional status, resident status, prior antibiotics prescriptions and medical history.

Other variables from the medical record that will be registered are length of stay, readmission, admission to intensive care unit, prescribed antibiotic treatment, in-hospital mortality, 30-day and 90-day mortality, Clostridium difficile infections and chest X-ray.

\section{Data monitoring}

The daily inclusion of participants will be monitored by the steering committee and the numbers of inclusion will be communicated every week to be emailed to the included centres. The primary analysis of data will be performed by the project assistants after the last patient has been included and all analyses performed. The results will be discussed and evaluated first in the steering committee and afterwards with all the included departments.

\section{Process auditing}

During data collection, an extern assessor will supervise the performance of all project assistants and an independent radiology expert will ensure data quality. Intraobservability on POC-US will be performed each month.

Overall risk for the participants in the randomised trial (POC-PCR sputum analysis) is minimal, as sputum collection is part of the standard care, and it will not affect the following diagnostic work-up. However, the POC-PCR results may inform the clinician in a favourable way before onset of patient treatment. Any protocol deviation and/or unknown/unexpected adverse event will be reported in REDCap, evaluated continuously by the steering committee, and reported to the treating physician and patient.

\section{Statistical analysis and plan}

According to the objectives, the study has been divided into substudies and for each, the primary and secondary outcomes, statistical analysis and sample size are presented.

\section{Objective 1: patient characteristics and treatment trajectory}

This substudy will include all participants. Patient characteristics associated to verified diagnosis will be presented with descriptive results, and logistic univariate and multivariate analysis will be carried out for selected risk indicators, including confounders in the final analysis. The primary outcome is the diagnosis of CAP and APN determined by the expert panel reference standard. Secondary outcomes are length of stay, 30-day mortality, in-hospital mortality, admission to intensive care unit and readmission to hospital within 30 days from day of discharge.

At least 10 variables have to be analysed, so at least 150 patients with a particular verified diagnosis are needed $(50+10$ events/variable).
Objective 2: diagnostic and prognostic accuracy of PCT and suPAR This diagnostic accuracy study will include all participants. Index tests are the concentration of CRP, PCT and suPAR. The expert panel is the reference standard. Diagnostic accuracy tests will be performed as primary analysis, where the test positive of the reference standard is the diagnosis of CAP, and of urinary tract infection. Secondary prognostic tests will be performed, using the reference standard of 30-day and 90-day mortality, in-hospital mortality, admission to intensive care, readmission to hospital within 30 days from day of discharge and length of stay.

The test positively cut-offs of the index tests will be determined exploratory by performing Youden index analysis to estimate the best cut-off. The CRP value will be available for the members of the expert panel, but the PCT and suPAR will not be available. The reference standard results will not be available for the index test performers.

A demographic characteristic of the study populations will be presented, and the time interval of the laboratory analysis of the biomarkers will be reported. Crosstabulation of the index test results by the reference standard results will be made including missing results, and used to determine diagnostic and prognostic accuracy expressed as sensitivity, specificity, predictive values and likelihood ratios reported with 95\% CIs where appropriate. Receiver operating characteristic (ROC) analysis will be performed. Statistical modelling will also be performed to explore the effect of combining tests on diagnostic accuracy in order to identify the most accurate diagnostic strategy.

The study is designed to be able to find a difference in area under the curve (AUC) from 0.7 to 0.8 between two tests, which requires 200 verified CAP cases and 200 controls (power 0.8, alpha 0.05, AUC below 0 hypothesis 0.7 ) and 150 verified pyelonephritis cases and 150 controls (power 0.8, alpha 0.05 , AUC below 0 hypothesis 0.6$).{ }^{42}$

\section{Objective 3: diagnostic accuracy of POC-UFC on diagnosing and excluding bacteriuria}

This diagnostic accuracy study will include all participants. Index test is the POC-UFC and reference standard is the urine culture. The primary outcome is bacteriuria, defined as significant growth of any bacteria. A urine culture will be considered positive with a cut-off of $>1000 \mathrm{CFU} / \mathrm{mL}$ for uropathogens and $>10.000 \mathrm{CFU} / \mathrm{mL}$ for others.

A secondary diagnostic test will be performed, where the reference standard is the expert panel assessment. The outcome is urinary tract infection. The test positive of the index test is bacteriuria combined with leucocytes.

The index test results will not be available for the performers of the reference standard test. The reference standard results will be available after the index test has been performed.

A demographic characteristic of the study populations will be presented. Cross-tabulation of the index test result by the reference standard results will be made including 
missing results, and used to determine diagnostic accuracy expressed as sensitivity, specificity, predictive values and likelihood ratios reported with 95\% CIs where appropriate. ROC analysis will also be performed.

Urine culture shows significant growth of uropathogenic bacterium in approximately $50 \%$ of people with suspected APN. ${ }^{25}$ Asymptomatic bacteriuria accounts for about $20 \%$ in the elderly population, depending on gender and age, ${ }^{43}$ which among 1000 inpatients suspected of infection, of which $15 \%$ have APN, gives a sensitivity of $50 \%$ (95\% CI: $42 \%$ to $58 \%)$ and a negative predictive value of $90 \%$ (95\% CI: $77 \%$ to $83 \%$ ). With the expectation of identifying at least 150 cases of APN among our study population, an improvement in sensitivity to $70 \%$ (95\% CI: $62 \%$ to $77 \%$ ) and negative predictive value to $95 \%$ (95\% CI: $93 \%$ to $96 \%$ ) could be found with $95 \%$ security.

\section{Objective 4: addition of POC-PCR analysis of sputum to the} diagnostic set-up for CAP on antibiotic prescribing

This randomised controlled trial will include all participants in track A, who had a sputum sample collected. Intervention group: sputum samples analysed by POCPCR. Control group: routine microbiology analysis. It is a superiority randomised trial. Primary outcome is targeted versus non-targeted antibiotic treatment prescribed at 4 hours after admission. Targeted treatment is defined as narrow-spectrum antibiotics directed against CAP, antibiotics directed against a detected respiratory pathogen or no antibiotics (eg, in the absence of a bacterial pathogen and/or presence of a viral pathogen) (online supplemental appendix VII). Non-targeted treatment is defined as broad-spectrum antibiotics not directed against a specific pathogen or antibiotics not directed against CAP. The analyses will follow the intention-to-treat principle and a hierarchical mixed-effects logistic model will be used to analyse the primary outcome to accommodate the hierarchical structure of the random effect, which manifests according to different personnel collecting the samples and geographical variation.

Secondary outcomes are length of stay, 30-day mortality, in-hospital mortality, admission to intensive care unit, readmission to hospital within 30 days from day of discharge and antibiotic treatment at 48 hours of admission. A reliability analysis for POC-PCR and routine culturing will be performed as secondary analysis calculating the intraclass correlation coefficient.

To achieve a power of $82 \%$ for the main analysis, 200 patients with suspected CAP must be included. To accommodate the bias presented by Gail et al, ${ }^{44}$ the generalised mixed-effects models will be adjusted for strong predictors. If the sample size is not sufficient for a generalised mixed-effects models, the corresponding univariate analysis will be conducted.

\section{Objective 5: diagnostic accuracy of POC-US and ULDCT on diagnosing CAP}

This diagnostic accuracy study will include all participants in track A, who had the HRCT performed. Index test is the POC-US, ULDCT and chest X-ray. The reference standard is HRCT. The primary outcome is inflammatory changes in the lungs compatible with CAP.

The index test results will not be available for the performers of the reference standard test. The reference standard results will be available after the index test has been performed.

A demographic characteristic of the study populations will be presented. Cross-tabulation of the index test results by the reference standard results will be made including missing results, and used to determine diagnostic accuracy expressed as sensitivity, specificity, predictive values and likelihood ratios reported with 95\% CIs where appropriate. ROC analysis will also be performed.

It is assumed that the reference standard will find $98 \%$ of the patients and index test $90 \%$. With a power of $80 \%$, at least 132 patients with verified CAP should be included (one-sided McNemar test).

\section{Objective 6: diagnostic accuracy of CEUS on diagnosing APN}

This diagnostic accuracy study will include all participants in track B, who had both the CEUS and MRI performed. Index test is the CEUS and reference standard is MRI. The primary outcome is the presence of renal inflammatory changes compatible with APN. The reference standard will be described by an expert radiologist, who before describing will be informed of some standardised clinical and paraclinical parameters (eg, fever, CRP, flank pain and relevant comorbidity), but will be blinded to the results of the other imaging investigations. The CEUS will be conducted and described by a consultant radiologist. The scans will be post-process evaluated in the software VueBox. Each kidney is divided into an upper, middle and lower part for each, and these regions are compared in the evaluation of diagnostic agreement.

The index test results will not be available for reference standard performer and describer. The reference standard results will not be available for the index test performers.

A demographic characteristic of the study populations will be presented, and the time interval of the two scans will be reported. Cross-tabulation of the index test result by the reference standard results will be made including missing results, and used to determine diagnostic accuracy expressed as sensitivity, specificity, predictive values and likelihood ratios reported with 95\% CIs where appropriate. ROC analysis will also be performed.

It is assumed that the reference standard finds $98 \%$ of patients and index test $90 \%$. With a power of $80 \%$, at least 132 patients must be included (one-sided McNemar test).

\section{Objective 7: diagnostic accuracy of POC-US in diagnosing}

hydronephrosis in patients suspected of APN

This diagnostic accuracy study will include all participants in track B, who had both the POC-US and MRI successfully conducted. Index test is the POC-US and reference standard is MRI. The primary outcome is the presence of hydronephrosis. The reference standard is described by 
an expert radiologist. The POC-US will be evaluated by the executive study assistants.

The index test results will not be available for reference standard evaluator. The reference standard results will not be available for the index test performers.

A demographic characteristic of the study populations will be presented, and the time interval of the two scans will be reported. Cross-tabulation of the index test result by the reference standard results will be made including missing results, and used to determine diagnostic accuracy expressed as sensitivity, specificity, predictive values and likelihood ratios reported with 95\% CIs where appropriate. ROC analysis will also be performed.

It is assumed that the reference standard finds $98 \%$ of patients and index test $90 \%$. With a power of $80 \%$, at least 132 patients must be included (one-sided McNemar test).

\section{Applicable to all substudies}

Annually, 5.7\% of patients admitted to an ED are diagnosed with CAP and $2.4 \%$ with APNs (data from the ED at Hospital Sønderjylland). Taking into account exclusion criteria, weekends/holidays/missing data, and experience in patient recruitment, it is estimated that at least 1000 patients admitted with suspected infection must be included in the study, of which at least 200 patients will be diagnosed with pneumonia and at least 150 patients with APN.

No interim analysis will be made. Non-participant analysis is performed. For missing data, multiple imputation is used. Any dropout during the study and the reason will be reported. It is anticipated that once the patients have consented, the dropout rate will be minimal.

\section{ETHICS AND DISSEMINATION}

The project was approved by the Regional Committees on Health Research Ethics for Southern Denmark (S-20200188), registered by the Danish Data Protection Agency (no. 20/60508) and by clinicaltrials.gov. Registration date was November-December 2020. Signed informed consent will be obtained from all participants after information of the project has been given both in writing and orally.

Participation in track A will contain additional imaging. Patients under the age of 40 years are therefore excluded from the CT due to the extra risk of developing cancer from the radiation. A local hospital physicist has helped with the following calculations: a typical HRCT gives a radiation dose of approximately $2.2 \mathrm{mSv}$ which corresponds to a cancer risk of 1:9100. An X-ray gives a radiation dose of approximately $0.06 \mathrm{mSv}$ which corresponds to a cancer risk of 1:333330. A ULDCT gives a radiation dose of approximately $0.1 \mathrm{mSv}$ which corresponds to a cancer risk of 1:200000. Participation in track A gives each participant approximately $2.26 \mathrm{mSv}$ (ULDCT and HRCT) which corresponds to a cancer risk of 1:8850. ${ }^{45-48}$ The examination time of ULDCT and HRCT is approximately 10 min.
Use of US contrast in rare cases causes allergic reactions; less than 1/10.000 exponents require medical treatment due to allergic reaction. ${ }^{49}$ The examination time of advanced US is approximately $20 \mathrm{~min}$.

MRI does not provide any radiation dose to the patients and is without intravenous contrast. The examination time is approximately $45 \mathrm{~min}$, which is aligned with normal MRI examination time.

Overall, risks related to participation in the study are considered minimal, and furthermore, chances are that the additional diagnostic imaging will inform the clinician in a favourable way before the onset of patient treatment.

The treating staff informs the patients about relevant test results. All medical records including laboratory and imaging can be assessed by the patient via the Danish public healthcare web portal (www.sundhed.dk).

\section{Protocol amendments}

Important protocol modifications like changes in eligibility criteria or outcome will be communicated to the relevant parties, that is, sponsor, trial registry and scientific ethical committee, and explicitly described in future publications.

\section{Dissemination policy}

The results of the study will be presented in English peerreviewed recognised scientific journals. The results of the project will also be disseminated through participation in academic and other conferences, as well as through the printed and electronic press. The author panel will include the steering committee, project assistants and local coordinators in accordance with the Vancouver criteria. No professional writers will be used. Positive, negative and inconclusive results will be published. Diagnostic accuracy studies will follow the STAndards for the Reporting Diagnostic accuracy studies,$^{50}$ cross-sectional studies will follow the guidelines for Strengthening the Reporting of Observational Studies in Epidemiology, ${ }^{51}$ and randomised studies will follow the Consolidated Standards of Reporting Trials. ${ }^{52}$

\section{Access to data}

Only the members of the steering committee and project assistants will have access to the final trial dataset. Other researchers may be granted access to the anonymised data for analysis on reasonable request to the corresponding authors.

\section{DISCUSSION}

COVID-19 and the consequent societal lockdown might affect trial recruitment and patient distribution. This might lead to an extended recruitment period, as patients suspected of an infection not related to COVID-19 may be admitted to other departments than the ED, so the ED will be able to handle the many patients with COVID19. The lockdown may also reduce the number of infections in the society, so fewer patients will visit the hospital, and the distribution of the infections might differ since, 
for example, the airborne-transmitted infections will be reduced. This challenge will, especially substudy 1 , be aware of when presenting the results.

After completion of the study, a novel diagnostic algorithm will be developed. Subsequently, the plan is to test the algorithm in a national setting including at least eight EDs. The results can be implemented in daily work and routines. The study will also be able to characterise the patients, who are diagnosed at the ED with an infection of unknown origin and prescribed broad-spectrum antibiotics.

The study is only generalisable to settings where appropriately trained staff and equipment can perform POCUS, and well-resourced settings where a rapid POC-PCR and POC-UFC service is available.

The results of the study will have both national and international interest, as the challenges are common and the solutions can easily be applied in hospitals with a similar technological context. Securing rapid and reliable diagnosis of two of the most common infections diagnosed in the ED will encourage the reduction of broad-spectrum antibiotics and thereby the development of multiresistant bacteria.

\section{Author affiliations}

${ }^{1}$ Emergency Department, University Hospital of Southern Denmark, Aabenraa, Denmark

${ }^{2}$ Department of Regional Health Research, University of Southern Denmark Faculty of Health Sciences, Odense, Denmark

${ }^{3}$ Department of Internal Medicine, University Hospital of Southern Denmark, Sønderborg, Denmark

${ }^{4}$ Department of Infectious Diseases, Odense University Hospital, Odense, Denmark ${ }^{5}$ Department of Clinical Research, University of Southern Denmark Faculty of Health Sciences, Odense, Denmark

${ }^{6}$ Department of Radiology, Odense University Hospital, Odense, Denmark

${ }^{7}$ Department of Clinical Microbiology, Odense University Hospital, Odense, Denmark

${ }^{8}$ Blood Samples, Biochemistry and Immunology, University Hospital of Southern Denmark, Aabenraa, Denmark

${ }^{9}$ Department of Clinical Microbiology, University Hospital of Southern Denmark, Vejle, Denmark

${ }^{10}$ Department of Respiratory Medicine, Odense University Hospital, Odense, Denmark

${ }^{11}$ Emergency Department, Odense University Hospital, Odense, Denmark

${ }^{12}$ Department of Microbiology, University Hospital of Southern Denmark, Aabenraa, Denmark

Acknowledgements In performing our protocol, we received help and guidance from some respected persons, who deserve our greatest gratitude: Research Radiographer Bo Mussmann from Radiology Department at Odense University Hospital in Denmark, Professor Michael Pedersen from Department of Clinical Medicine at Aarhus University Hospital in Denmark, Professor Ivan Brandslund from Department of Clinical Biochemistry at Hospital Lillebælt in Denmark, Statistician Andreas Kristian Pedersen from Department of Research and Learning at University Hospital of Southern Denmark, and Research Assistant Mette Bach Nielsen from Emergency Department at University Hospital of Southern Denmark.

Collaborators Steering committee: composed of representatives from the involved type of departments: emergency, microbiology, biochemistry and radiology. The role of the committee is to develop the scientific framework of the study, and make final decisions on major issues during the data collection and data management period. The committee is responsible for all financial issues. Members of the steering committee are: HS-A, OG, FSR, ERBP and CBM. Roles and responsibilities: University Hospital of Southern Denmark is the legal sponsor; CBM is the study chief investigator (Christian.Backer.Mogensen@rsyd.dk) and HS-A is the principal investigator.
Contributors HS-A, OG, FSR, ERBP, CØ, CBL, TAS, SP, MBC and CBM conceptualised and all authors designed the study and data collection in detail. HS-A, AH, MHL, MBC and CBM reviewed the literature. AH, MHL, MBC and MAAH will recruit participants. HS-A, OG, FSR, ERBP, CØ, CBL, TAS, SP, MBC and CBM will supervise data collection and analysis. $\mathrm{HS}-\mathrm{A}, \mathrm{AH}, \mathrm{MHL}, \mathrm{MBC}$, MAAH and CBM will carry out statistical analysis and write the first manuscripts, which will be critically reviewed by all authors, who will finally approve the manuscripts before submission. HS-A and CBM are responsible for the overall content as guarantors.

Funding This work was supported by the Region of Southern Denmark (Damhaven 12, 7100 Vejle, Denmark; kontakt@rsyd.dk) (grant number A583), University of Southern Denmark (Campusvej 55, 5230 0dense, Denmark; sdu@sdu.dk) (grant numbers 16/44667 and 17/10636), and Hospital Sønderjylland (Kresten Philipsensvej 15, 6200 Aabenraa, Denmark, email shs.kontakt@rsyd.dk) (grant numbers 20/20505and 21/9582).

Disclaimer The financial sponsors had no influence on the data, analysis, results or content of publication.

Competing interests None declared.

Patient consent for publication Obtained.

Provenance and peer review Not commissioned; externally peer reviewed.

Supplemental material This content has been supplied by the author(s). It has not been vetted by BMJ Publishing Group Limited (BMJ) and may not have been peer-reviewed. Any opinions or recommendations discussed are solely those of the author(s) and are not endorsed by BMJ. BMJ disclaims all liability and responsibility arising from any reliance placed on the content. Where the content includes any translated material, BMJ does not warrant the accuracy and reliability of the translations (including but not limited to local regulations, clinical guidelines, terminology, drug names and drug dosages), and is not responsible for any error and/or omissions arising from translation and adaptation or otherwise.

Open access This is an open access article distributed in accordance with the Creative Commons Attribution Non Commercial (CC BY-NC 4.0) license, which permits others to distribute, remix, adapt, build upon this work non-commercially, and license their derivative works on different terms, provided the original work is properly cited, appropriate credit is given, any changes made indicated, and the use is non-commercial. See: http://creativecommons.org/licenses/by-nc/4.0/.

\section{ORCID iDs}

Helene Skjøt-Arkil http://orcid.org/0000-0002-2236-6375

Mathias Amdi Hertz http://orcid.org/0000-0002-8064-7642

Christian Backer Mogensen http://orcid.org/0000-0002-8254-089X

\section{REFERENCES}

1 WHO. Antimicrobial resistance - Global report and surveillance. France: World Health Organization, 2014.

2 Bager FE-I J, Larsen AR, Sönksen UW. DANMAP 2018 - Use of antimicrobial agents and occurrence of antimicrobial resistance in bacteria from food animals, food and humans in Denmark, 2018.

3 Skjøt-Arkil H, Mogensen CB, Lassen AT, et al. Carrier prevalence and risk factors for colonisation of multiresistant bacteria in Danish emergency departments: a cross-sectional survey. BMJ Open 2019;9:e029000.

4 Sundhedsstyrelsen. Vejledning Om ordination AF antibiotika. Copenhagen: Danish Health Organisaztion, 2012.

5 Sundhedsstyrelsen. Vejledning Om forebyggelse AF spredning AF MRSA, 2016.

6 Sundhedsstyrelsen. Vejledning OG forebyggelse AF Om spredning AF CPO, 2018.

7 ÆEldreministeriet S-o. National handlingsplan for antibiotika til mennesker - tre målbare mål for en reduktion af antibiotikaforbruget frem mod 2020, 2017.

8 Hellesøe AM, Anhøj CB.;, Jensen J.; . LKT Antibiotika afs/utnings- OG evalueringsrapport, 2019.

9 Cartuliares MB, Sundal LM, Gustavsson S, et al. Limited value of sputum culture to guide antibiotic treatment in a Danish emergency department. Dan Med J 2020;67:A11190641.

10 Funk DJ, Kumar A. Antimicrobial therapy for life-threatening infections: speed is life. Crit Care Clin 2011;27:53-76.

11 World Health Organization. The top 10 causes of death, 2018. Available: https://www.who.int/news-room/fact-sheets/detail/thetop-10-causes-of-death [Accessed July 2019].

12 Kennedy M, Joyce N, Howell MD, et al. Identifying infected emergency department patients admitted to the hospital ward at risk 
of clinical deterioration and intensive care unit transfer. Acad Emerg Med 2010;17:1080-5.

13 Chandra A, Nicks B, Maniago E, et al. A multicenter analysis of the ED diagnosis of pneumonia. Am J Emerg Med 2010;28:862-5.

14 Reed WW, Byrd GS, Gates RH, et al. Sputum gram's stain in community-acquired pneumococcal pneumonia. A meta-analysis. West J Med 1996;165:197-204.

15 Schuetz P, Christ-Crain M, Müller B. Procalcitonin and other biomarkers to improve assessment and antibiotic stewardship in infections--hope for hype? Swiss Med Wkly 2009;139:318-26.

16 Savvateeva EN, Rubina AY, Gryadunov DA. Biomarkers of community-acquired pneumonia: a key to disease diagnosis and management. Biomed Res Int 2019;2019:1-20.

17 Hey J, Thompson-Leduc P, Kirson NY, et al. Procalcitonin guidance in patients with lower respiratory tract infections: a systematic review and meta-analysis. Clin Chem Lab Med 2018;56:1200-9.

18 Self WH, Balk RA, Grijalva CG, et al. Procalcitonin as a marker of etiology in adults hospitalized with community-acquired pneumonia. Clin Infect Dis 2017;65:183-90.

19 Hamie L, Daoud G, Nemer G, et al. SuPAR, an emerging biomarker in kidney and inflammatory diseases. Postgrad Med J 2018;94:517-24.

20 Masajtis-Zagajewska A, Nowicki M. New markers of urinary tract infection. Clin Chim Acta 2017:471:286-91.

21 Caterino JM, Leininger R, Kline DM, et al. Accuracy of current diagnostic criteria for acute bacterial infection in older adults in the emergency department. J Am Geriatr Soc 2017;65:1802-9.

22 Bourcier J-E, Paquet J, Seinger M, et al. Performance comparison of lung ultrasound and chest X-ray for the diagnosis of pneumonia in the ED. Am J Emerg Med 2014;32:115-8.

23 Orso D, Guglielmo N, Copetti R. Lung ultrasound in diagnosing pneumonia in the emergency department: a systematic review and meta-analysis. Eur J Emerg Med 2018;25:312-21.

24 Claeys KC, Blanco N, Morgan DJ, et al. Advances and challenges in the diagnosis and treatment of urinary tract infections: the need for diagnostic stewardship. Curr Infect Dis Rep 2019;21:11.

25 Shallcross L, Gaskell K, Fox-Lewis A, et al. Mismatch between suspected pyelonephritis and microbiological diagnosis: a cohort study from a UK teaching hospital. J Hosp Infect 2018;98:219-22.

26 Quaia E, Correas JM, Mehta M, et al. Gray scale ultrasound, color Doppler ultrasound, and contrast-enhanced ultrasound in renal parenchymal diseases. Ultrasound Q 2018;34:250-67.

27 Mitterberger M, Pinggera GM, Colleselli D, et al. Acute pyelonephritis: comparison of diagnosis with computed tomography and contrastenhanced ultrasonography. BJU Int 2008;101:341-4.

28 Kazmierski B, Deurdulian C, Tchelepi $\mathrm{H}$, et al. Applications of contrast-enhanced ultrasound in the kidney. Abdom Radiol 2018;43:880-98.

29 Huang H-S, Tsai C-L, Chang J, et al. Multiplex PCR system for the rapid diagnosis of respiratory virus infection: systematic review and meta-analysis. Clin Microbiol Infect 2018;24:1055-63.

30 Long B, Koyfman A. The emergency department diagnosis and management of urinary tract infection. Emerg Med Clin North Am 2018;36:685-710.

31 Rowe TA, Juthani-Mehta M. Diagnosis and management of urinary tract infection in older adults. Infect Dis Clin North Am 2014;28:75-89.

32 Institut SS. Urinvejsinfektioner: Blærebetændelse OG nyrebækkenbetændelse Statens serum Institut: Statens Seum
Institut, 2017. Available: https://www.ssi.dk/sygdomme-beredskabog-forskning/sygdomsleksikon/u/urinvejsinfektioner [Accessed April 2019].

33 Herráez O, Asencio MA, Carranza R, et al. Sysmex UF-1000i flow cytometer to screen urinary tract infections: the URISCAM multicentre study. Lett Appl Microbiol 2018;66:175-81.

34 Chan A-W, Tetzlaff JM, Altman DG, et al. Spirit 2013 statement: defining standard protocol items for clinical trials. Ann Intern Med 2013;158:200-7.

35 The region of southern Denmark traw. Den Regionale Antibiotikagruppe. Diagnostik OG behandling AF akutte infektioner på sygehusene I region Syddanmark, 2016.

36 Skovsted TA, Petersen ERB, Fruekilde M-B, et al. Validation of suPAR turbidimetric assay on Cobas $\AA$ (c502 and c702) and comparison to suPAR ELISA. Scand J Clin Lab Invest 2020;80:327-35.

37 BioFire. Filmarray pneumonia panel instruction booklet RFITASY0144/145. UT: BioFire SLC, 2018.

38 Harris PA, Taylor R, Thielke R, et al. Research electronic data capture (REDCap)--a metadata-driven methodology and workflow process for providing translational research informatics support. J Biomed Inform 2009;42:377-81.

39 Volpicelli G, Elbarbary M, Blaivas M, et al. International evidencebased recommendations for point-of-care lung ultrasound. Intensive Care Med 2012;38:577-91.

40 Laursen CB, Clive A, Hallifax R, et al. European respiratory Society statement on thoracic ultrasound. Eur Respir J 2021;57:2001519.

41 Laursen BLG, Davidsen JR, et. al. Basal klinisk ultralydsdiagnostik. Copenhagen: Munksgaard, 2017. https://basal-kliniskultralydsdiagnostik.munksgaard.dk

42 Biosoft. easyROC: a web-tool for ROC curve analysis. Available: http://www.biosoft.hacettepe.edu.tr/easyROC/ [Accessed 03 Jan 2020].

43 Mody L, Juthani-Mehta M. Urinary tract infections in older women: a clinical review, JAMA 2014:311:844-54

44 GAIL MH, WIEAND S, PIANTADOSI S. Biased estimates of treatment effect in randomized experiments with nonlinear regressions and omitted covariates. Biometrika 1984;71:431-44.

45 Huda W, Ogden KM, Khorasani MR. Converting dose-length product to effective dose at CT. Radiology 2008;248:995-1003.

46 Sodickson A, Baeyens PF, Andriole KP, et al. Recurrent CT, cumulative radiation exposure, and associated radiation-induced cancer risks from CT of adults. Radiology 2009;251:175-84.

47 Brenner DJ, Hall EJ. Computed tomography--an increasing source of radiation exposure. N Engl J Med 2007;357:2277-84.

48 Mayo-Smith WW, Hara AK, Mahesh M, et al. How I do it: managing radiation dose in CT. Radiology 2014;273:657-72.

49 Chong WK, Papadopoulou V, Dayton PA. Imaging with ultrasound contrast agents: current status and future. Abdom Radiol 2018;43:762-72.

50 Cohen JF, Korevaar DA, Altman DG, et al. Stard 2015 guidelines for reporting diagnostic accuracy studies: explanation and elaboration. BMJ Open 2016;6:e012799.

51 Vandenbroucke JP, von Elm E, Altman DG, et al. Strengthening the reporting of observational studies in epidemiology (STROBE): explanation and elaboration. PLOS Med 2007;4:e297.

52 Moher D, Hopewell S, Schulz KF, et al. Consort 2010 explanation and elaboration: updated guidelines for reporting parallel group randomised trials. BMJ 2010;340:c869. 